\title{
Calreticulin promotes proliferation and extracellular matrix expression through Notch pathway in cardiac fibroblasts
}

\author{
Xiaoying Fan ${ }^{* B, D, F}$, Yuan Yao*C,D,F, Yao Zhang ${ }^{A, D-F}$ \\ Department of Cardiovascular Medicine, $2^{\text {nd }}$ Affiliated Hospital of Harbin Medical University, China \\ A - research concept and design; $B$ - collection and/or assembly of data; $C$ - data analysis and interpretation; \\ $\mathrm{D}$ - writing the article; $\mathrm{E}$ - critical revision of the article; $\mathrm{F}$ - final approval of the article
}

\section{Address for correspondence \\ Yao Zhang \\ E-mail:zhangya0159@126.com \\ Funding sources \\ None declared \\ Conflict of interest \\ None declared \\ * The first 2 authors contributed equally to the work.}

Received on April 20, 2017

Reviewed on May 17, 2017

Accepted on June 5, 2017

DOI

10.17219/acem/74430

\section{Copyright}

Copyright by Author(s)

This is an article distributed under the terms of the

Creative Commons Attribution Non-Commercial License

(http://creativecommons.org/licenses/by-nc-nd/4.0/)

\begin{abstract}
Background. Cardiac fibrosis is one of the most important underlying causes of several cardiac diseases. The role of calreticulin (CRT) in cardiac diseases has already been established. The over- or under-expression of CRT can lead to cardiac diseases.

Objectives. This study was aimed to explore the effect of CRT on cardiac fibrosis and also to investigate the possible underlying molecular mechanism.

Material and methods. Human cardiac fibroblast cells (HCF) were used in the experiment. The cells were transfected with the CRT expression vector constructed by sub-cloning the full-length wild-type CRT coding sequence into pcDNA3.1 (pc-CRT group), empty construct pcDNA3.1 (pcDNA3.1 group), CRT-specific siRNA (si-CRT), and si-NC (negative control). The Cell Counting Kit-8 (CCK-8) assay, apoptosis assay and invasion assay were performed. Quantitative real time polymerase chain reaction (GRT PCR) and western blot analysis were performed to measure the expressions of different mRNAs and proteins.
\end{abstract}

Results. The CRT expression was significantly increased $(p<0.01)$ and decreased $(p<0.01)$ in the $p c-C R T$ and si-CRT groups, respectively. The CRT over-expression led to increased cell viability and invasiveness $(p<0.05)$ and a decreased percentage of apoptotic cells. The over-expression of CRT led to a significant increase in the expressions of collagen (I and III) $(p<0.01)$ and matrix metalloproteinases (MMP-2 and 9 ) $(p<0.05)$. The Notch pathway was also significantly activated $(p<0.05)$ by the over-expression of CRT and vice versa when suppressed.

Conclusions. The results showed that the CRT over-expression was associated with increased cell viability and invasiveness and decreased apoptosis, and the activation of the Notch pathway in HCF, which suggests its possible implication in CRT-induced cardiac fibrosis.

Key words: calreticulin, Notch pathway, cardiac fibroblasts, extracellular matrix, proliferation 


\section{Introduction}

Cardiac fibrosis is a pathophysiological condition characterized by the accumulation of extracellular matrix in the myocardium and is considered one of the most important factors implicated in various cardiac diseases. ${ }^{1,2}$ The regenerative capacity of the adult mammalian heart is negligible, and therefore fibrosis occurs in cardiac diseases associated with acute death of cardiomyocytes. ${ }^{1-4}$ In acute myocardial infarction (AMI), sudden death of a large number of cardiomyocytes leads to the triggering of inflammatory pathways and dead cell replacement with collagen-based scar tissue formation. ${ }^{5,6}$ Other than the acute onset of cardiac diseases like AMI, several other chronic cardiac diseases are associated with insidious accumulation of collagen in the interstitial and perivascular regions. ${ }^{6}$ Aging is another factor that leads to the development of cardiac fibrosis and ultimately to diastolic failure. ${ }^{1}$ Several long-standing cardiac diseases, like chronic hypertension and aortic stenosis, might also lead to widespread cardiac fibrosis, associated with loss of cardiac contractility and diastolic dysfunction, which ultimately leads to both systolic and diastolic heart failure because of persistent pressure overload. ${ }^{4}$ Besides pressure overload, volume overload, as in the valvular regurgitant type of cardiac lesions, can also lead to cardiac fibrosis due to the deposit of a large amount of non-collagenous matrix. ${ }^{4}$ Other common causes of cardiac fibrosis include alcoholism, anthracycline use, metabolic disturbances like diabetes, obesity, etc. ${ }^{7-9}$

Calreticulin (CRT) is a $\mathrm{Ca} 2+$ binding protein which acts as a chaperone in the storage of $\mathrm{Ca} 2+$ and the maintenance of $\mathrm{Ca} 2+$ homeostasis. ${ }^{10}$ Calreticulin is widely expressed in the embryonic heart; however, its expression is downregulated following birth. ${ }^{11}$ Kypreou et al. have explored the role of altered expression of CRT in the fibrosis of several organs, such as the lung, heart and renal epithelial cells. Although the over-expression of CRT could be implicated in lung and renal epithelial cell fibrosis, there were no changes in the expression of CRT in the cardiac model. ${ }^{12}$ Only a few studies have been conducted to explore the implications of the CRT over-expression in certain cardiac conditions, namely diastolic dysfunction, ventricular dilation, cardiac arrhythmia, and sudden cardiac arrest. ${ }^{10-14}$

In this study, we have explored the effect of CRT on the proliferation and extracellular matrix expression in cardiac fibroblasts and also investigated the possible underlying mechanisms.

\section{Material and methods}

\section{Cell culture}

Human cardiac fibroblasts (HCF) were purchased from the Cell Bank of Tongpai Biotechnology Co., Ltd. (Shanghai, China). The base medium for the HCF cell line was formulated Dulbecco's Modified Eagle's Medium (DMEM; Gibco Inc., Thermo Fisher, Waltham, USA). To make the complete growth medium, we added the following components to the base medium: $4.5 \mathrm{~g} / \mathrm{L}$ glucose and fetal bovine serum to a final concentration of $10 \%$. Exponentially growing cultures were maintained in a humidified atmosphere of $5 \%$ carbon dioxide $\left(\mathrm{CO}_{2}\right)$ at $37^{\circ} \mathrm{C}$.

\section{Plasmids and siRNA transfection}

The CRT expression vector (pcDNA3.1-CRT) was constructed by sub-cloning the full-length wild-type CRT coding sequence into pcDNA3.1, and was then confirmed by sequencing. The empty construct pcDNA3.1 was transfected as a control. The target sequence for CRT-specific siRNA was 5'-GGAGCAGUUUCUGGACGGA-3', synthesized by GenePharma Co. (Shanghai, China). Cell transfections were conducted using Lipofectamine 3000 reagent (Invitrogen, Carlsbad, USA), following the manufacturer's protocol.

\section{Cell Counting Kit-8 assay}

Human cardiac fibroblasts were seeded in a 96-well plate with 5000 cells/well, and then transfected by the plasmids or siRNAs for $48 \mathrm{~h}$. Cell proliferation was assessed by CCK-8 (Dojindo Molecular Technologies, Gaithersburg, USA). Briefly, after stimulation, the CCK-8 solution was added to the culture medium, and the cultures were incubated for $1 \mathrm{~h}$ at $37^{\circ} \mathrm{C}$ in humidified $95 \%$ air and $5 \% \mathrm{CO}_{2}$. The absorbance was measured at $450 \mathrm{~nm}$, using a Microplate Reader (Bio-Rad, Hercules, USA).

\section{Apoptosis assay}

Cell apoptosis analysis was performed using propidium iodide (PI) and fluorescein isothiocynate (FITC)-conjugated Annexin V staining (Invitrogen, Grand Island, USA). Briefly, the cells were washed in phosphate buffered saline (PBS) and fixed in 70\% ethanol. The fixed cells were then washed twice in PBS and stained in PI/FITC-Annexin $\mathrm{V}$ in the presence of $50 \mu \mathrm{g} / \mathrm{mL}$ RNase A (Sigma-Aldrich, St. Louis, USA), and then incubated for $1 \mathrm{~h}$ at room temperature in the dark. Flow cytometry analysis was done by using a FACScan (Beckman Coulter, Fullerton, USA). The data was analyzed using FlowJo software (Ashland, USA).

\section{Invasion assay}

The invasion behavior of HCF was determined using 24-well Millicell Hanging Cell Culture inserts with $8 \mathrm{~mm}$ PET membranes (Millipore, Bedford, USA). Briefly, after the cells were treated for the indicated condition, $5.0 \times 10^{4} \mathrm{HCF}$ in $200 \mu \mathrm{L}$ serum-free DMEM were plated onto BD BioCoat ${ }^{\mathrm{TM}}$ Matrigel $^{\mathrm{TM}}$ invasion chambers $(8 \mu \mathrm{M}$ pore size polycarbonate filters; BD Biosciences, San Jose, 
USA), while the complete medium, containing 10\% FBS was added to the lower chamber. After processing the invasion chambers for $48 \mathrm{~h}\left(37^{\circ} \mathrm{C}, 5 \% \mathrm{CO}_{2}\right)$ in accordance with the manufacturer's protocol, the non-invading cells were removed with a cotton swab; the invading cells were fixed in $100 \%$ methanol, and then stained with crystal violet solution and counted microscopically. The data is presented as the average number of cells attached to the bottom surface from 5 randomly chosen fields.

\section{Quantitative real time polymerase chain reaction}

Total RNA was isolated from transfected cells by using TRIzol reagent (Invitrogen) and treated with DNaseI (Promega, Madison, USA). Reverse transcription was performed by using the Multiscribe RT kit (Applied Biosystems, Foster City, USA) and random hexamers or oligo(dT). The reverse transcription conditions were $10 \mathrm{~min}$ at $25^{\circ} \mathrm{C}, 30 \mathrm{~min}$ at $48^{\circ} \mathrm{C}$, and a final step of $5 \mathrm{~min}$ at $95^{\circ} \mathrm{C}$. The sequences of the primers were as follows: CRT-forward, 5'-CGGGGTACCCGCCACCATGGCGATGCTGCTATCCGTGCCG-3'; reverse, 5'-CCGGAATTCCAGCTCGTCCTTGGCCTG-3', GAPDH-forward, 5'-GCACCGTCAAGGCTGAGAAC'-3; reverse, 5'-TGGTGAAGACGCCAGTGGA-3'.

\section{Western blot}

The protein used for western blotting was extracted using RIPA lysis buffer (Beyotime Biotechnology, Shanghai, China) supplemented with protease inhibitors (Roche, Guangzhou, China). The proteins were quantified using the $\mathrm{BCA}^{\mathrm{TM}}$ Protein Assay Kit (Pierce, Appleton, USA). The western blot system was established using the Bio-Rad Bis-Tris Gel system according to the manufacturer's instructions. Rabbit-anti-mouse CRT antibody was purchased from Biotechnology, Inc. (Santa Cruz, USA). Glyceraldehyde 3-phosphate dehydrogenase (GAPDH) antibody was purchased from Sigma-Aldrich. The primary antibodies were prepared in 5\% blocking buffer at a dilution of 1:1,000. The primary antibody was incubated with the membrane at $4^{\circ} \mathrm{C}$ overnight, followed by washing and incubation with a secondary antibody marked by horseradish peroxidase for $1 \mathrm{~h}$ at room temperature. After rinsing, the polyvinylidene difluoride (PVDF) membrane carrying blots and antibodies was transferred into the BioRad ChemiDoc ${ }^{\mathrm{TM}}$ XRS system, and then $200 \mu \mathrm{L}$ Immobilon Western Chemiluminescent HRP Substrate (Millipore) was added to cover the membrane surface. The signals were captured and the intensity of the bands was quantified using Image Lab ${ }^{\text {TM }}$ Software (Bio-Rad).

\section{Statistical analysis}

All experiments were repeated 3 times. The results of multiple experiments are presented as mean \pm standard deviation (SD). Statistical analyses were performed using SPSS 19.0 statistical software (IBM, Armonk, USA). P-values were calculated using one-way analysis of variance (ANOVA). A p-value of $<0.05$ was considered to indicate a statistically significant result.

\section{Results}

\section{Expression of calreticulin in different groups of human cardiac fibroblasts}

The expression of CRT was measured in different groups of HCF cells by qRT-PCR. Human cardiac fibroblasts were transfected with a vector constructed by sub-cloning the full-length wild-type CRT coding sequence into pcDNA3.1. The pc-CRT cells showed a significant increase $(\mathrm{p}<0.01)$ in the expression of CRT compared to the control group (pcDNA3.1 group) transfected with empty construct (Fig. 1A). Western blot analysis also revealed increased expression of CRT in the pc-CRT group (Fig. 1B). Similarly, mRNA expression of CRT in HCF transfected with CRTspecific siRNA, as in the si-CRT group, was significantly suppressed $(\mathrm{p}<0.01)$ compared to the control group (si-NC group) (Fig. 1C). Western blot analysis also supported the above finding (Fig. 1D).

\section{Effects of calreticulin expression on cell viability}

The assessment of cell viability in different groups of HCF for 4 days revealed that the over-expression of CRT, as in the pc-CRT group, promoted cell viability, whereas the silencing of CRT expression in the si-CRT group of HCF suppressed it (Fig. 2).

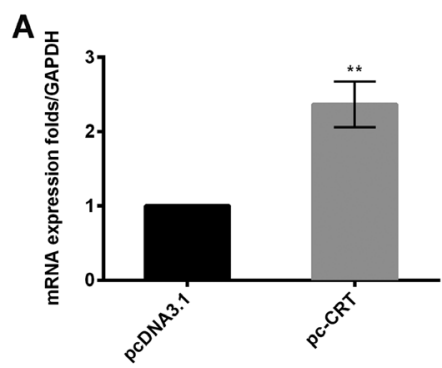

B
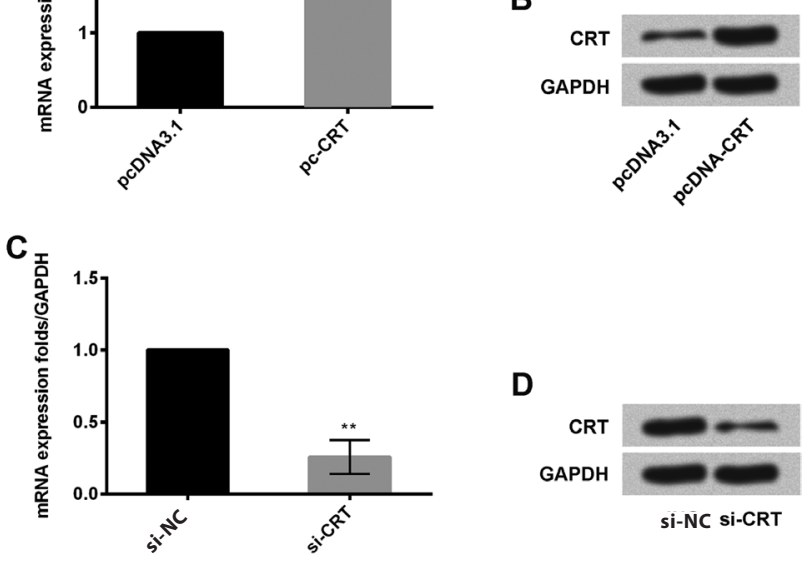

D

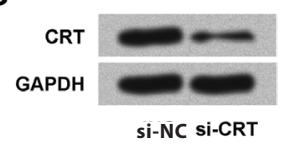

Fig. 1. Expression of CRT in different groups of HCF measured by qRT-PCR analysis $(A, C)$ and western blot analysis (B,D)

CRT - calreticulin; GAPDH - glyceraldehyde 3-phosphate dehydrogenase; qRT-PCR - quantitative real time polymerase chain reaction; ${ }^{* *} p<0.01$. 


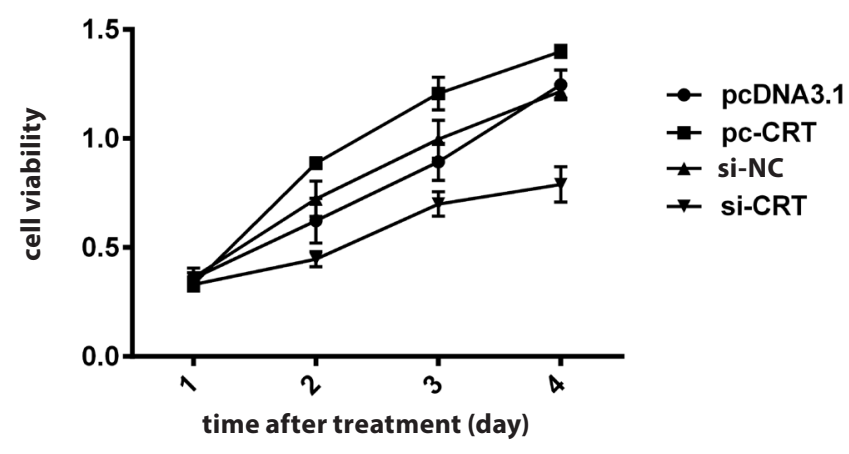

Fig. 2. Effects of CRT expression (both over- and under-expression) on cell viability

CRT - calreticulin.

\section{Effect of calreticulin expression on apoptosis}

The apoptosis assay revealed that although the over-expression of CRT, as in the pc-CRT group, showed no significant change in apoptotic cells as compared to the control group (pcDNA3.1 group), the down-regulation of CRT, as in the si-CRT group, revealed a significant increase in cell apoptosis compared to the si-NC group (Fig. 3).

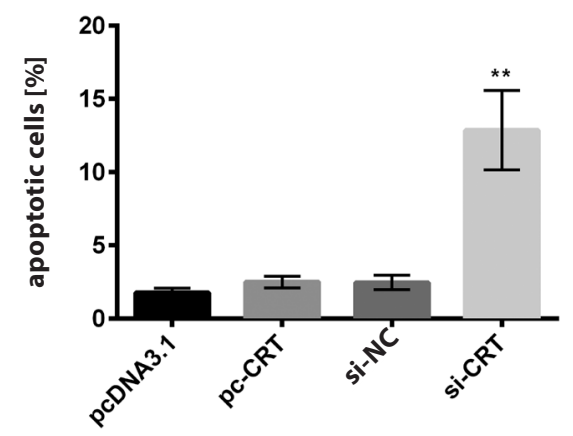

Fig. 3. Effects of CRT expression (both over- and under-expression) on the percentage of apoptosis cells

CRT - calreticulin; ** $p<0.01$.

\section{Effect of calreticulin expression on cellular invasiveness}

The over-expression of CRT, as in the pc-CRT group, revealed a significant increase $(\mathrm{p}<0.05)$ in cellular invasiveness compared to the control group (pcDNA3.1 group), whereas the suppression of the CRT expression, as in the si-CRT group, revealed a significant decrease $(\mathrm{p}<0.05)$ in cellular invasiveness compared to the control group, si-NC (Fig. 4).

\section{Effects of calreticulin expression on the expression of collagen and matrix metalloproteinases}

Quantitative real time polymerase chain reaction revealed that collagen I, collagen III, metalloproteinases

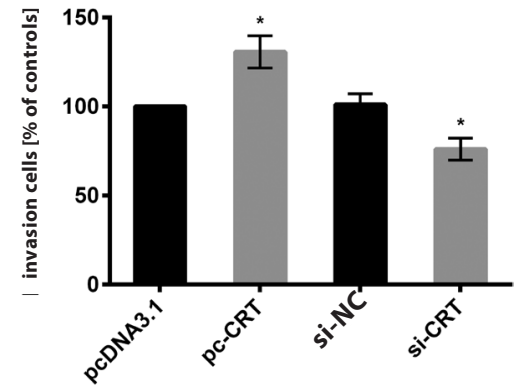

Fig. 4. Effects of CRT expression (both over- and under-expression) on cell invasiveness

CRT - calreticulin; * $p<0.05$

(MMPs) MMP-2 and MMP-9 mRNAs were significantly expressed $(\mathrm{p}<0.01, \mathrm{p}<0.01, \mathrm{p}<0.05$, and $\mathrm{p}<0.05$, respectively) in HCF overexpressing CRT, as in the pc-CRT group compared to the control group (Fig. 5A). Similarly, silencing of CRT expressions, as in the si-CRT group, led to decreased, although not significantly, expressions of collagen I, collagen III, MMP-2, and MMP-9 mRNAs compared to the control group (Fig. 5A). Western blot analysis also revealed that the expression levels of collagen I, collagen III, MMP-2, and MMP-9 proteins were decreased in the si-CRT group and increased in the pc-CRT group (Fig. 5B).
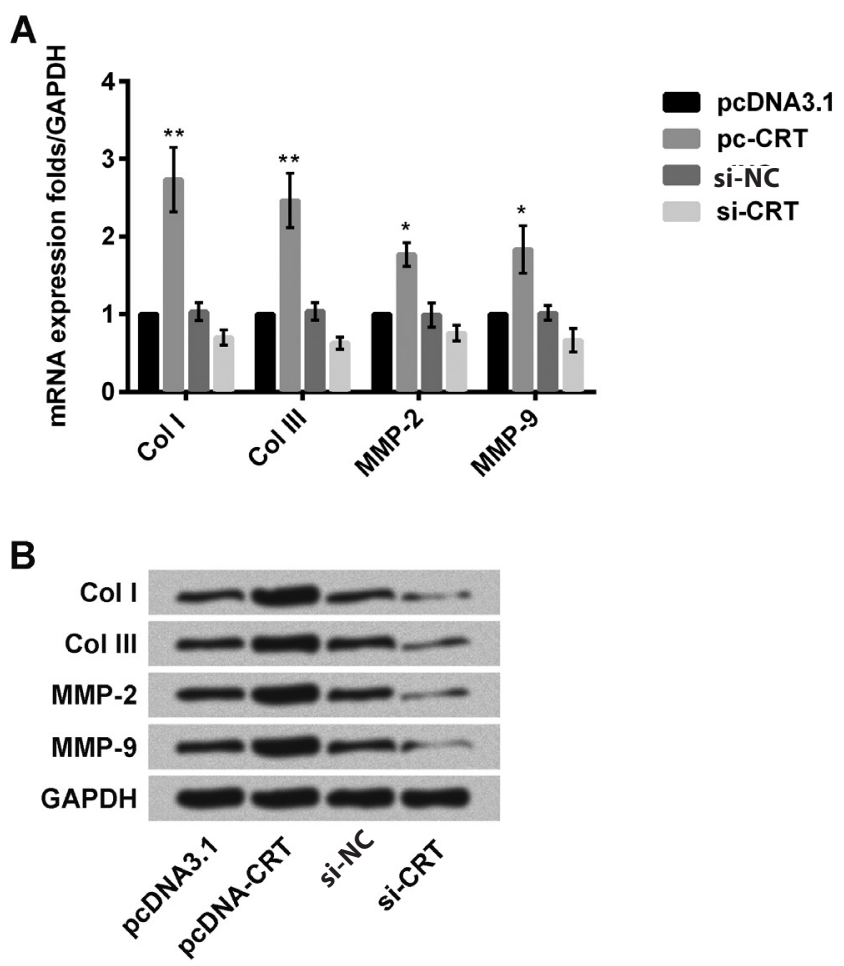

Fig. 5. Effects of CRT expression (both over- and under-expression) on the expression of collagen (I and III) and metalloproteinases (MMP-2 and MMP-9) measured by qRT-PCR analysis (A) and western blot analysis (B)

CRT - calreticulin; GAPDH - glyceraldehyde 3-phosphate dehydrogenase; qRT-PCR - quantitative real time polymerase chain reaction; * $p<0.05$; ** $p<0.01$. 


\section{Effect of calreticulin expression on the Notch pathway}

The over-expression of CRT, as in the pc-CRT group, revealed a significant increase $(p<0.05)$ in the expression levels of Notch 1, 2 and 3 receptors (Fig. 6A). Again, HCF with the silencing of the CRT expression, as in the si-CRT group, revealed decreased expression levels of the 3 abovementioned Notch receptors with a significant suppression $(\mathrm{p}<0.05)$ of the expression of Notch 2 in the si-CRT group (Fig. 6A). Western blot analysis also revealed the same results; the expression levels of Notch 1, 2 and 3 receptors were increased and decreased in the pc-CRT and si-CRT groups, respectively (Fig. 6B).
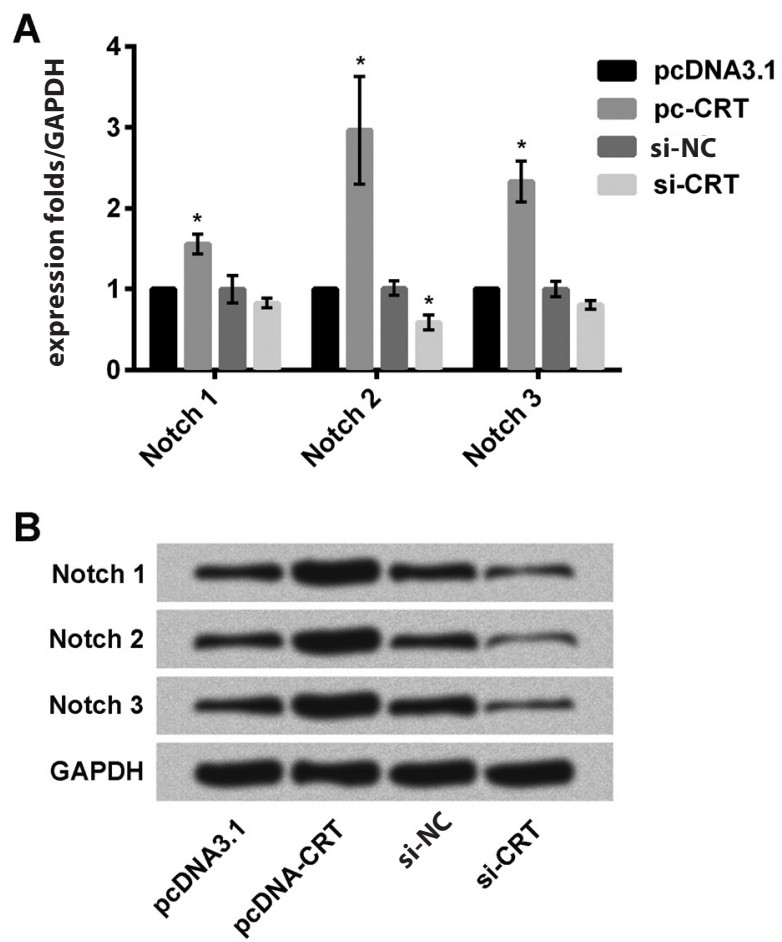

Fig. 6. Effect of CRT expression (both over- and under-expression) on the Notch pathway measured by qRT-PCR analysis (A) and western blot analysis (B)

CRT - calreticulin; GAPDH - glyceraldehyde 3-phosphate dehydrogenase; qRT-PCR - quantitative real time polymerase chain reaction; ${ }^{*} p<0.05$.

\section{Discussion}

Cardiovascular diseases are one of the leading causes of mortality worldwide. ${ }^{15}$ Like in the healing process of any other part of the body, cardiac injury is also healed by scar tissue formation. Scar tissue formation is mediated mainly by activating fibroblasts, which secrete collagen and ultimately produce myocardial fibrosis. ${ }^{16}$ Fibrosis is very important in enhancing the mechanical stability of the cardiac wall to prevent its rupture following injury. However, it can, unfortunately, disrupt electrical coupling among the surrounding myocytes. ${ }^{17}$
In a healthy heart, fibroblasts play an important role in maintaining the integrity of the myocardial structure. These are one of the predominant cell types in the heart besides myocytes, which in turn exceed the myocytes in number but not in volume. ${ }^{16}$ Fibroblasts act as the sentinel cells, which in turn maintain the balance between the synthesis and degradation of collagen and other components of the extracellular matrix. Myocardial injury triggers differentiation of these fibroblasts into myofibroblasts, thus facilitating the process of wound healing. ${ }^{16}$

Several studies have already established the role of CRT in the pathogenesis of different diseases, like cancer and cardiovascular disease. ${ }^{18-20}$ Although it has already been established that CRT is critical for cardiac development, its role in cardiac disease remains elusive. In spite of demonstrating the deleterious effects on the heart, following the exposure to the over-expression of CRT, it has also been established that CRT is essential for the development of a normal heart. ${ }^{19}$ Hence, it is said that balanced expression of CRT is essential for proper development and adequate functioning of the heart, and both the over- and under-expression of CRT can be deleterious to the heart. ${ }^{19}$

In this study, we have explored the effect of CRT on HCF and also its possible underlying mechanism.

Following the transfection of HCF, the expressions of CRT mRNA and protein were measured. The CRT expression was significantly increased $(p<0.01)$ both in terms of mRNA and protein expression in the pc-CRT group compared to controls (pcDNA3.1 group) (Fig. 1A,1B). Similarly, the CRT expression was significantly suppressed $(\mathrm{p}<0.01)$ in the si-CRT group (Fig. 1C,1D).

Similar to our findings, several studies have established that the over-expression of CRT was associated with high prevalence of cardiac fibrosis. ${ }^{19,20}$

We also found out that the over-expression of CRT, as in the pc-CRT group, was associated with increased cell viability (Fig. 2) and invasiveness ( $<$ 0.05) (Fig. 4), and the suppression of apoptosis (Fig. 3), whereas the knockdown of the CRT expression, as in the si-CRT group, showed just the opposite results (Fig. 2-4). Again, both qRT-PCR and western blot analysis demonstrated that the over-expression of CRT was associated with a significant increase in the expression levels of collagen and MMPs ( $\mathrm{p}<0.01$ and $\mathrm{p}<0.05$ ), respectively (Fig. 5A,5B).

Similar to our findings, Groenendyk et al. also demonstrated that the over-expression of CRT was associated with increased cardiac fibrosis. ${ }^{21}$

Studies have already implicated the over-expression of the Notch pathway in cardiac fibrosis. ${ }^{22}$ In this study, we demonstrated that the over-expression of CRT was associated with the activation of the Notch pathway (Fig. 6A, 6B).

It can be concluded that the over-expression of CRT is associated with increased expression of collagen and MMPs, and also the activation of the Notch pathway in HCF, which suggests its possible implication in cardiac fibrosis. Hence, CRT can be considered as a novel target for the treatment of cardiac fibrosis. 


\section{References}

1. Kong $\mathrm{P}$, Christia $\mathrm{P}$, Frangogiannis NG. The pathogenesis of cardiac fibrosis. Cell Mol Life Sci. 2014;71:549-574.

2. Berk BC, Fujiwara K, Lehoux S. ECM remodeling in hypertensive heart disease. J Clin Invest. 2007;117:568-575.

3. Frangogiannis NG. Regulation of the inflammatory response in cardiac repair. Circ Res. 2012;110:159-173.

4. Borer JS, Truter S, Herrold EM, et al. Myocardial fibrosis in chronic aortic regurgitation molecular and cellular responses to volume overload. Circulation. 2002;105:1837.

5. Ashrafian H, Mckenna WJ, Watkins H. Disease pathways and novel therapeutic targets in hypertrophic cardiomyopathy. Circ Res. 2011; 109:86-96.

6. Kania G, Blyszczuk P, Eriksson U. Mechanisms of cardiac fibrosis in inflammatory heart disease. Trends Cardiovasc Med. 2009;19:247.

7. Bernaba BN, Chan JB, Lai CK, Fishbein MC. Pathology of late-onset anthracycline cardiomyopathy. Cardiovasc Pathol. 2010;19:308-311.

8. Asbun J, Villarreal FJ. The pathogenesis of myocardial fibrosis in the setting of diabetic cardiomyopathy. J Am Coll Cardiol. 2006;47:693-700.

9. Bharati S, Lev M. Cardiac conduction system involvement in sudden death of obese young people. Am Heart J. 1995;129:273-281.

10. Michalak M, Groenendyk J, Szabo E, Gold LI, Opas M. Calreticulin, a multi-process calcium-buffering chaperone of the endoplasmic reticulum. Biochem J. 2009;417:651.

11. Mesaeli N, Nakamura K, Zvaritch E, et al. Calreticulin is essential for cardiac development. J Cell Biol. 1999;144:857-868.
12. Kypreou KP, Kavvadas $\mathrm{P}$, Karamessinis $\mathrm{P}$, et al. Altered expression of calreticulin during the development of fibrosis. Proteomics. 2008;8: 2407-2419.

13. Hattori K, Nakamura K, Hisatomi Y, et al. Arrhythmia induced by spatiotemporal overexpression of calreticulin in the heart. Mol Genet Metab. 2007;91:285-293.

14. Nakamura K, Robertson M, Liu G, et al. Complete heart block and sudden death in mice overexpressing calreticulin.J Clin Invest. 2001;107: 1245-1253.

15. Miragoli M, Salvarani N, Rohr S. Myofibroblasts induce ectopic activity in cardiac tissue. Circ Res. 2007;101:755.

16. Nguyen TP, Qu Z, Weiss JN. Cardiac fibrosis and arrhythmogenesis: The road to repair is paved with perils. J Mol Cell Cardiol. 2014;70:83-91.

17. Vasquez C, Mohandas P, Louie KL, Benamer N, Bapat AC, Morley GE. Enhanced fibroblast-myocyte interactions in response to cardiac injury. Circ Res. 2010;107:1011.

18. Lu YC, Weng WC, Lee H. Functional roles of calreticulin in cancer biology. Biomed Res Int. 2015;2015:1-9.

19. Martinho-Dias D, Leite-Moreira A, Castro-Chaves P. Calreticulin in the heart: From embryological development to cardiac pathology. Curr Mol Med. 2015;16:112-119.

20. Lee $D, O k a T$, Hunter $B$, et al. Calreticulin induces dilated cardiomyopathy. PLOS ONE. 2013;8:e56387-e56387.

21. Groenendyk J, Lee D, Jung J, et al. Inhibition of the unfolded protein response mechanism prevents cardiac fibrosis. PLOS ONE. 2016;11: e0159682.

22. Collesi C, Zentilin L, Sinagra G, Giacca M. Notch1 signaling stimulates proliferation of immature cardiomyocytes.J Cell Biol. 2008;183:117-128. 